\title{
Clinical and pathological response to pre-operative crizotinib in a patient with ALK-translocated NSCLC
}

Chiara Catania1,*, Massimo Barberis ${ }^{2}$, Francesco Petrella ${ }^{3}$, Piergiorgio Solli ${ }^{3}$, Tommaso Martino De Pas ${ }^{4}$, Giuseppe Perrone ${ }^{5}$, Spitaleri Gianluca ${ }^{1}$, Cristina Noberasco ${ }^{1}$, Antonio Passaro ${ }^{1}$, Elena Guerini Rocco ${ }^{2}$ and Filippo de Marinis ${ }^{1}$

${ }^{1}$ Division of Thoracic Oncology, European Institute of Oncology (IEO), Milan, Italy

${ }^{2}$ Clinic Unit of Histopathology and Molecular Diagnostics: European Institute of Oncology, Milan, Italy

${ }^{3}$ Department of Thoracic Surgery, European Institute of Oncology, Milan, Italy

${ }^{4}$ Oncology Unit of Thymic cancer, Rare Tumors and Sarcomas, Milan, Italy

${ }^{5}$ Division of Molecolar Diagnostic and Citogenetic: Policlinico Universitario Campus Bio-Medico Rome, Italy

\begin{abstract}
A 65-year-old non-smoker female was diagnosed with lung adenocarcinoma clinically staged as IV M1a because of bilateral pulmonary lesions. After a differential response to chemotherapy, further analyses allowed us to re-stage the tumor as a synchronous bilateral local disease with unilateral ALK (Anaplastic lymphoma kinase) rearrangement. Combined treatment with chemotherapy, crizotinib and surgery, with clinical and pathological tumor-response to pre-operative crizotinib, obtained complete tumors remission, and the patient is still disease free after 11 months since the last tumor resection. As far as we know this is the first report of a clinical and pathological regression of an early-stage ALK-rearranged NSCLC treated with neo-adjuvant crizotinib. This report supports further studies to assess activity and efficacy of ALK-inhibitors in neoadjuvant setting.
\end{abstract}

Keywords: crizotinib; NSCLC; neo-adjuvant treatment; anaplastic lymphoma kinase; surgery

\section{Introduction}

Patients with locally advanced non-small cell lung cancer (stage IIIA N2) usually undergo neoadjuvant chemotherapy in order to reduce the tumor size, address micrometastatic disease and improve clinical outcome. In these patients, a standard platinum-based treatment results in a pathological downstaging in 40\%-60\% [1-4] of the cases and a pathological complete response in 5\%-10\% [5], but the effect on the overall survival is still modest, with an estimated benefit of $4 \%$ to $8 \%$ at 5 years. Favourable prognostic factors for survival are complete pathological response and downstaging of mediastinal lymph nodes in the resection specimen. Therefore more active agents given as induction treatment could improve the clinical outcome of patients with locally advanced disease. In the last year, epidermal growth factor receptor (EGFR) tyrosine-kinase inhibitors (TKI) and tyrosine kinase inhibitor (TKI) of ALK have demonstrated considerable activity for the cure of the specific subgroup patients with tumors harboring EGFR mutations or ALK translocations. There is limited evidence for use of EGFR targeted therapy in a neoadjuvant setting and very limited case of use of ALK targeted therapies in a neoadjuvant setting. Rearrangements of the ALK gene are present in 3\% to $5 \%$ of non-small cell lung cancers (NSCLCs) [6, 7]. They define a distinct subgroup of NSCLC that typically occurs in younger patients, never smoked and that has adenocarcinoma histologic characteristics
[8-10]. Crizotinib is an oral small-molecule tyrosine kinase inhibitor (TKI) of ALK, MET, and ROS1 kinases [11]. In phase 1 and 2 studies, crizotinib treatment resulted in objective tumor responses in approximately $60 \%$ of patients with ALK-positive NSCLC and in progression-free survival of 7 to 10 months [12-14]. In a randomized phase 3 trial involving patients with advanced ALK-positive NSCLC who had received previous platinum-based chemotherapy, crizotinib was superior to single-agent second-line chemotherapy with either pemetrexed or docetaxel [15]. PROFILE 1014 study compared crizotinib vs chemotherapy in 343 patients ALK-positive who had no previous treatment for advanced NSCLC. They were randomized

*Corresponding author: Chiara Catania, MD, Division of Thoracic Oncology, European Institute of Oncology (IEO), Via G. Ripamonti 435, 20141 Milan, Italy. Email: chiara.catania@ieo.it

Received 27 July 2016 Revised 10 October 2016 Accepted 19 October 2016 Published 28 October 2016

Citation: Catania C, Barberis M, Petrella F, Solli P, DePas TM, Perrone G, Gianluca S, Noberasco C, Passaro A, Rocco EG, deMarinis F. Clinical and pathological response to pre-operative crizotinib in a patient with ALKtranslocated NSCLC.J Cancer Res Ther. 2016; 4(6):55-58. DOI: 10.14312/20524994.2016-10

Copyright: (c) 2016 Catania C, et al. Published by NobleResearch Publishers This is an open-access article distributed under the terms of the Creative Commons Attribution License, which permits unrestricted use, distribution and reproduction in any medium, provided the original author and source are credited. 
to either receive crizotinib vs pemetrexed plus platinum. Progression-free survival for crizotinib group $(n=172)$ was 10.9 months and for chemotherapy group $(n=171)$ was 7.0 months. The overall response rate (ORR) was $74 \%$ (95 $\%$ Cl 67-81) for crizotinib group vs $45 \%$ (95 \% Cl 37-53) for chemotherapy $(P<0.001)$ [16]. Crizotinib was approved for ALK positive, locally advanced, and metastatic NSCLC. However, there has been no data about the preoperative crizotinib treatment in NSCLC patients. We report the case of a 45-year-old female who underwent surgical resection after neo-adjuvant treatment with crizotinib. The patient presented with what was thought initially to be stage IV non-small cell lung cancer involving both lungs but she was then re-staged as having two localized primary bilateral lung cancers rather than metastatic malignancy.

\section{Case report}

We present the case of a 65-year-old non-smoker female who was diagnosed in July 2013 with bilateral lung tumors. Presentingsymptomswereamoderatedyspneaonexertion, anorexia and asthenia. A total body CT-scan showed a left perihilar and a right lower lobe lesion together with many small bilateral nodules and ground glass opacities. A 18fluordeoxyglucose (FDG) positron emission tomography (PET) showed pathologic accumulations of the radiotracer in the area of parenchymal thickening in the left lower lung and in many further areas at the posterior basal segment of the right lower lobe. Left bronchial biopsies showed a TTF1-positive p40-negative adenocarcinoma with ALK genetic rearrangements (FISH analysis Abbott Vysis breakapart probe) in $25 \%$ of the tumor cells (Figure 1 ).

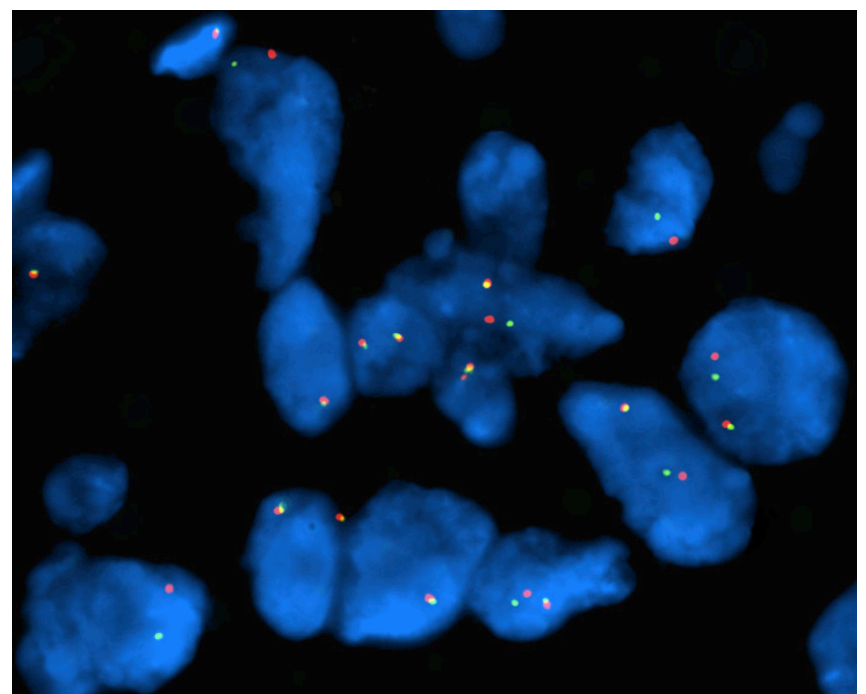

Figure 1 ALK genetic rearrangements (FISH analysis Abbott Vysis breakapart probe) in $25 \%$ of the tumor cells.

According to a clinical tumor stage IV M1a (7th TNM), being ALK-inhibitors not approved in Italy for first-line treatment, the patient received systemic chemotherapy with cisplatin and pemetrexed. A Response Evaluation Criteria in Solid Tumors (RECIST) stable disease (minor tumor reduction) of the left lesion together with a slight progression of the larger contralateral lesions were observed in a CT-scan evaluation performed after three cycles. According to this differential response, we performed a biopsy of the major right lung lesion. A different histology was found: well- differentiated adenocarcinoma with a bronchioloalveolar pattern of growth, without ALK rearrangement, EGFR wild type. In the light of the finding of two different lung cancers with different molecular patterns, the multidisciplinary team decided to surgically manage the ALK-negative righttumor, unresponsive to systemic chemotherapy, with the aim of giving a subsequent ALK-inhibitor for treating the left residual ALK-positive tumor. On January 2014, the patient underwent video-thoracoscopic right lower lobectomy and radical lymphadenectomy. Pathological report described a 3,3 cm sub-pleural TTF-1 positive invasive adenocarcinoma with prevalent lepidic growth (Figures 2 and 3) without any other cancer lesions in the whole lobe. The tumor was therefore staged ypT2aypN0. No EGFR (exon 18,19,20 and 21) and KRAS gene (codon 12, 13 and 61) mutations were found by Sanger bi-directional sequencing. FISH analysis for ALK rearrangement (Abbott Vysis break- apart probe) was negative. From February to August 2014 the patient received crizotinib $250 \mathrm{mg}$ twice daily, with no relevant toxicity.

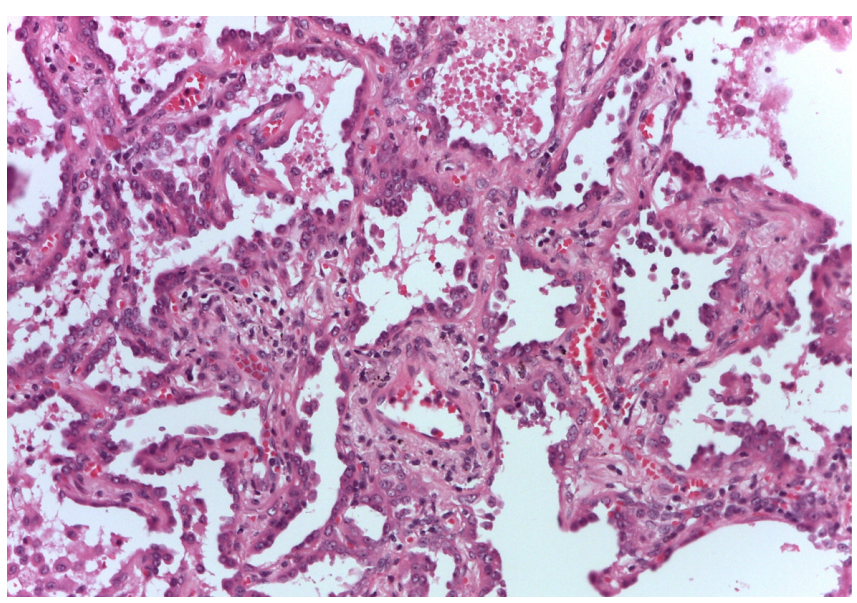

Figure 2 Pathological report described a 3,3 cm sub-pleural TTF-1 positive invasive denocarcinoma with prevalent lepidic growth.

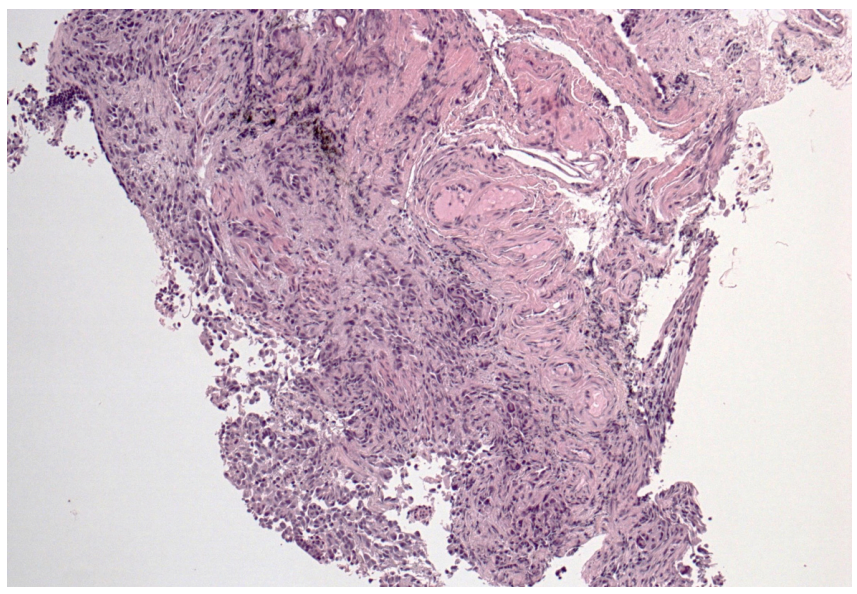

Figure 3 Left bronchial biopsies showed a TTF1-positive p40-negative adenocarcinoma.

A subsequent FDG-PET tumor reassessment showed a clinical response of the residual left tumor, with tumor reduction and a lower tumor uptake (Figure 4). Therefore, after 6 months of crizotinib administration, on august 2014 the patient underwent a left lower lobectomy and radical lymphadenectomy with curative intent with any complications during and following surgery. Histological 
report showed a solid 2,2 cm sub-pleural tumor prevalently made by poorly cellulated fibrous tissue containing rare acinar structures lined by atypical glandular cells. Scarred fibrosis was the predominant morphological finding. Only at the periphery of the lesion areas of adenocarcinoma with lepidic growth could be seen (Figures 5 and 6). Aggregates of foamy macrophages were present in the central areas of the tumor. Tumor cells strongly expressed TTF-1. The atypical glandular structures within the scarred fibrosis expressed ALK fusion protein (Figure 7); more than 30\% of the tumor nuclei showed ALK rearrangement at FISH analysis. After 11 months of follow up, the patient is still disease free.

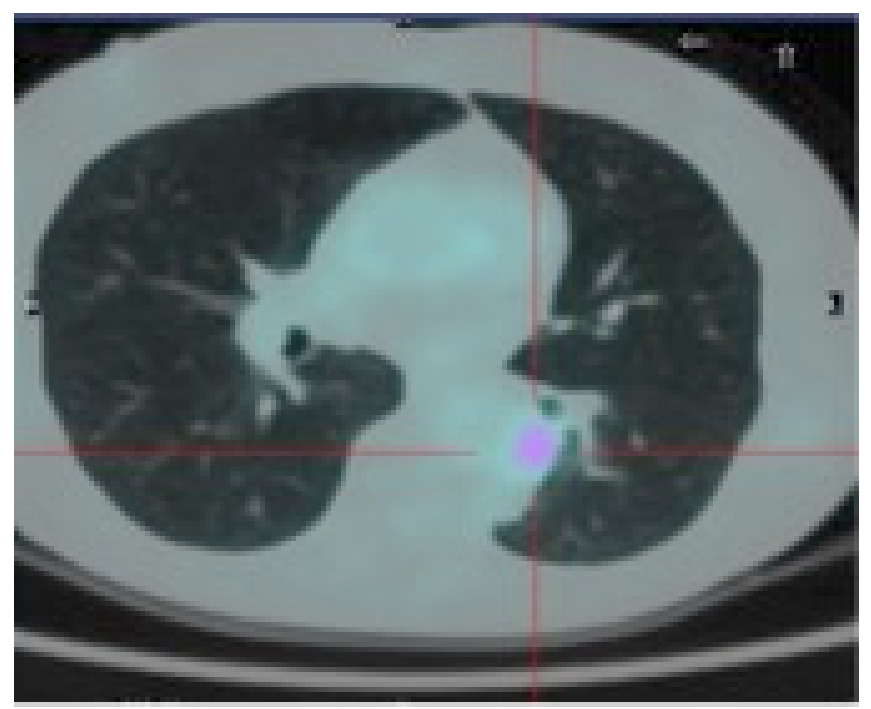

Figure 4a Pre crizotinib: FDG-PET tumor reassessment.

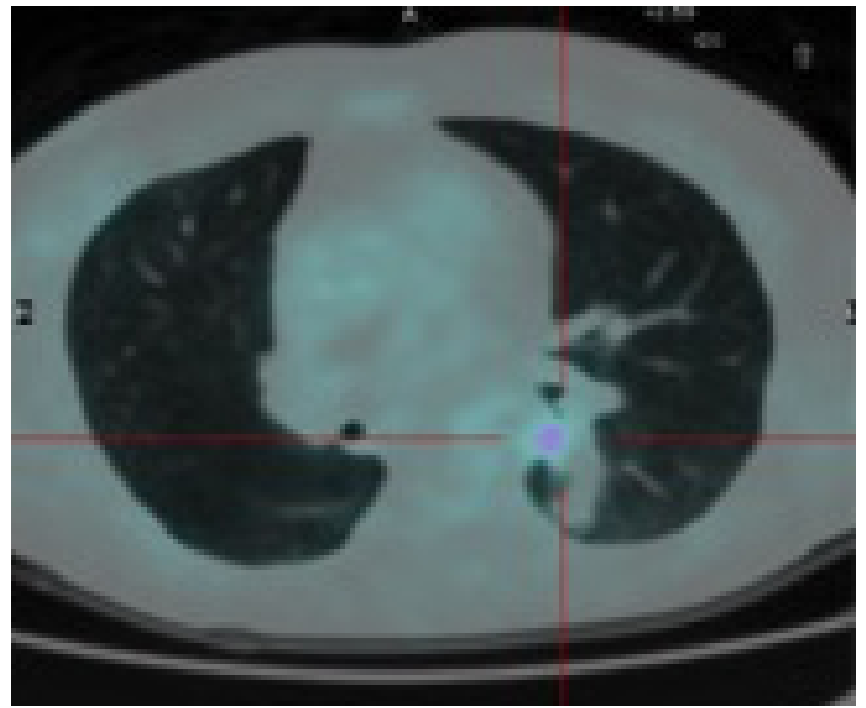

Figure 4b Post crizotinib: FDG-PET tumor reassessment showed a clinical response of the residual left tumor, with tumor reduction and a lower tumor uptake.

\section{Discussion}

Target therapy has changed the prognosis of patients with NSCLC harboring druggable molecular targets, such as ALK rearrangement, being more effective than chemotherapy with regards to both overall response rate and progression-free survival $[17,18]$. Missing recognition of these targets could strongly affect the patient's outcome.

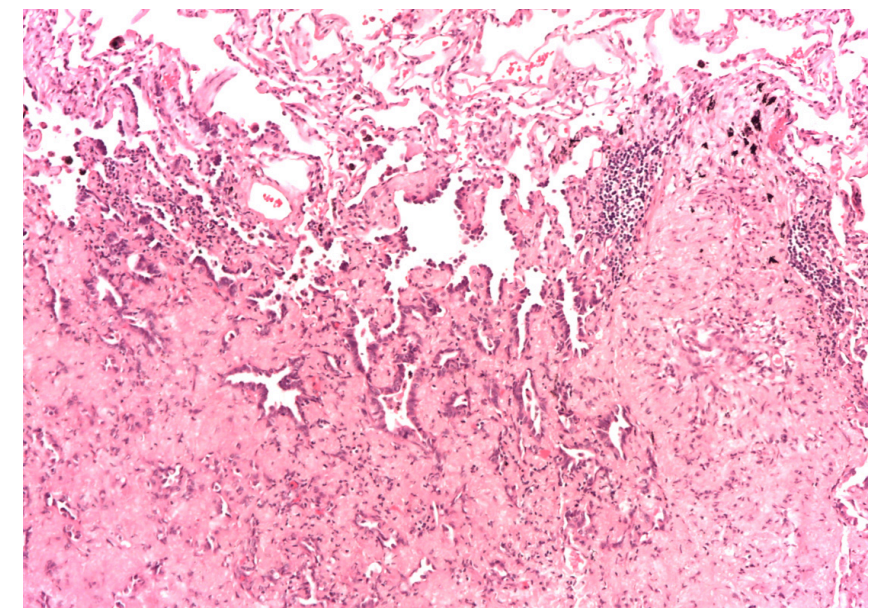

Figure 5 After 6 months of crizotinib administration; sub-pleural tumor prevalently made by poorly cellulated fibrous tissue containing rare acinar structures lined by atypical glandular cells. Scarred fibrosis was the predominant morphological finding. Only at the periphery of the lesion areas of adenocarcinoma with lepidic growth could be seen.

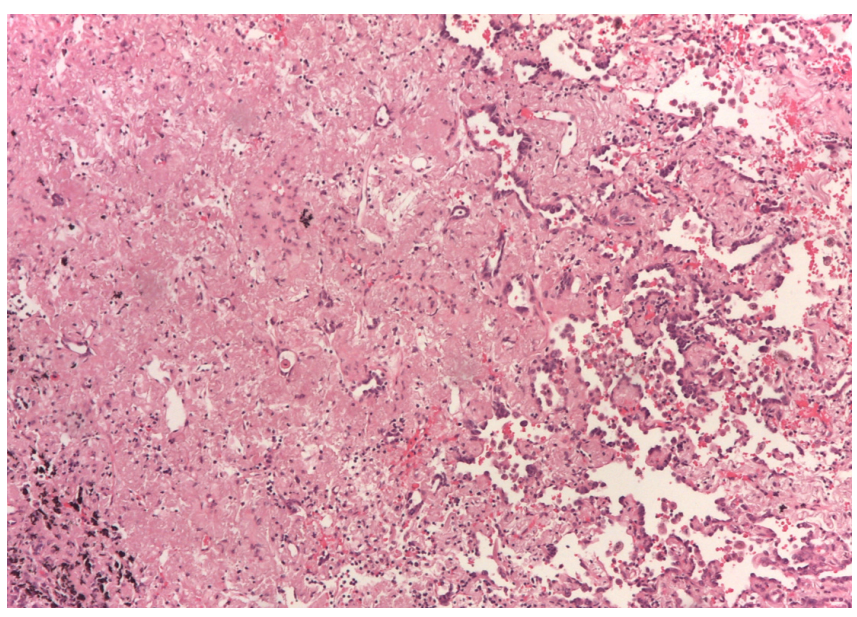

Figure 6 After 6 months of crizotinib administration; sub-pleural tumor prevalently made by poorly cellulated fibrous tissue containing rare acinar structures lined by atypical glandular cells. Scarred fibrosis was the predominant morphological finding. Only at the periphery of the lesion areas of adenocarcinoma with lepidic growth could be seen.

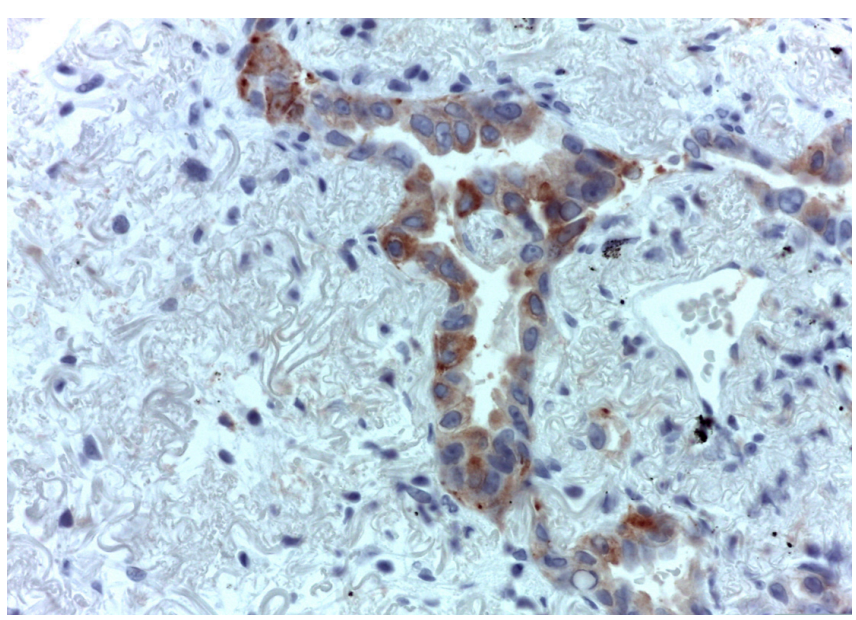

Figure 7 Aggregates of foamy macrophages were present in the central areas of the tumor. Tumor cells strongly expressed TTF-1. The atypica glandular structures within the scarred fibrosis expressed ALK fusion protein.

An interesting aspect of this case was the ability of the multidisciplinary team to understand that the patient was affected by two different tumors, with different 
molecular profiles, which led to a restaging from M1a to two synchronous primary tumors supporting a curative bilateral surgical approach. Furthermore, we underline the major activity of pre-operative crizotinib observed in this case. At present, although crizotinib showed a major and sustained antitumor activity in patients with advanced ALK-rearranged NSCLC, its role as a pre-operative systemic treatment has not been adequately investigated. As a matter of fact, molecular profiling of early stage NSCLC is not advised by international guidelines and the current standard of induction treatment is chemotherapy i.e., according to the last European Society for Medical Oncology (ESMO) guidelines, neo-adjuvant regimens with at least three cycles of platinum-based chemotherapy should be administered. Notably, favorable prognostic factors for survival in the subset of patients treated with induction chemotherapy are a complete pathological response and/or down-staging of mediastinal lymph nodes in the resection specimen. In this case, despite only 6-months of crizotinib administration, we obtained both a radiological response and a pathological evidence of major signs of tumor regression, with no tumor relapse after an 11 -months follow up. The development of target therapy offers a luxury option to improve the outcome of patients with curable advanced NSCLC, but there are still few studies exploring this opportunity. Eva E. Schaake et al., evaluated tumor response to 3 weeks of neo-adjuvant erlotinib administration in an enriched population (never-smoker, female sex, nons-quamous histology, or Asian ethnicity) of 60 patients with early-stage NSCLC. At surgery, pathologic examination showed more than $50 \%$ necrosis in $23 \%$ patients, of whom $5 \%$ had more than $95 \%$ tumor necrosis. This pilot experience demonstrated that this tyrosine kinase inhibitor has good activity in a neo-adjuvant setting [19]. Consistently, EGFR-TKI activity was reported also in short course of pre-operative treatment in stage I-II EGFR mutated NSCLC. Lara- Guerra et al. reported a 100\% tumor reduction in 6 patients with clinical stage I EGFR-mutated NSCLC treated with 28-days cycle of gefitinib. Rizvi NA and colleagues reported an $81 \%$ RECIST tumor response in 18 pts with EGFR-mutated stage I-II NSCLC after a 21-days gefitinib administration [20].

There is a paucity of information in regards to crizotinib as neo-adjuvant therapy for NSCLC and this case report could be of benefit to the medical community. There is only one published case report presented a patient with EML4/ ALK fusion gene-positive lung cancer who was treated with ALK inhibitor followed by surgery [21]. Favorable prognostic factors for survival are complete pathological response and down-staging of mediastinal lymph nodes in the resection specimen and more active agents given as induction treatment could improve the clinical outcome of patients with locally advanced disease. We report a clinical and pathological regression of an early-stage ALKrearranged NSCLC treated with neo-adjuvant crizotinib.

\section{Conclusion}

This case supports prospective studies to assess activity and efficacy of ALK-inhibitors in neoadjuvant setting in patients with ALK-rearranged, locally advanced NSCLC, evaluating both the rate of complete pathological response/ tumor downstaging and the patients outcome.

\section{Conflicts of interest}

The authors wish to express that they have no conflicts of interest.

\section{References}

[1] Betticher DC, Hsu Schmitz SF, Tötsch M, Hansen E, Joss C, et al. Mediastinal lymph node clearance after docetaxel-cisplatin neoadjuvant chemotherapy is prognostic of survival in patients with stage IIIA pN2 non-small-cell lung cancer: A multicenter phase II trial. J Clin Oncol. 2003; 21(9):1752-1759.

[2] Van Zandwijk N, Smit EF, Kramer GW, Schramel F, Gans S, et al. Gemcitabine and cisplatin as induction regimen for patients with biopsy-proven stage IIIA N2 non-small-cell lung cancer: A phase II study of the European Organization for Research and Treatment of Cancer Lung Cancer Cooperative Group (EORTC 08955). J Clin Oncol. 2000; 18(14):2658-2664.

[3] Van Meerbeeck JP, Kramer GW, Van Schil PE, Legrand C, Smit EF, et al. Randomized controlled trial of resection versus radiotherapy after induction chemotherapy in stage IIIA-N2 non-small-cell lung cancer. J Natl Cancer Inst. 2007: 99(6):442-450.

[4] Martin J, Ginsberg RJ, Venkatraman ES, Bains MS, Downey RJ, et al. Long-term results of combined-modality therapy in resectable nonsmall-cell lung cancer. J Clin Oncol. 2002; 20(8):1989-1995.

[5] Mouillet G, Monnet E, Milleron B, Puyraveau M, Quoix E, et al. Pathologic complete response to preoperative chemotherapy predicts cure in early-stage non-small-cell lung cancer: Combined analysis of two IFCT randomized trials. J Thorac Oncol. 2012; 7(5):841-849.

[6] Soda M, Choi YL, Enomoto M, Takada S, Yamashita Y, et al. Identification of the transforming EML4-ALK fusion gene in non-small-cell lung cancer. Nature. 2007; 448(7153):561-566.

[7] Rikova K, Guo A, Zeng Q, Possemato A, Yu J, et al. Global survey of phosphotyrosine signaling identifies oncogenic kinases in lung cancer. Cell. 2007; 131(6):1190-1203.

[8] Shaw AT, Yeap BY, Mino-Kenudson M, Digumarthy SR, Costa DB, et al. Clinical features and outcome of patients with non-small-cell lung cancer who harbor EML4-ALK. J Clin Oncol. 2009; 27(26):4247-4253.

[9] Camidge DR, Doebele RC. Treating ALK-positive lung cancer-early successes and future challenges. Nat Rev Clin Oncol. 20123; 9(5):268277.

[10] Blackhall FH, Peters S, Bubendorf L, Dafni U, Kerr KM, et al. Prevalence and clinical outcomes for patients with ALK-positive resected stage I-III adenocarcinoma: results from the European Thoracic Oncology Platform Lungscape Project. J Clin Oncol. 2014; 32(25):2780-2787.

[11] Christensen JG, Zou HY, Arango ME, Li Q, Lee JH, et al. Cytoreductive antitumor activity of PF-2341066, a novel inhibitor of anaplastic lymphoma kinase and c-Met, in experimental models of anaplastic large-cell lymphoma. Mol Cancer Ther. 2007; 6(12 Pt 1):3314-3322.

[12] Kwak EL, Bang YJ, Camidge DR, Shaw AT, Solomon B, et al. Anaplastic lymphoma kinase inhibition in non-small-cell lung cancer. N Engl J Med. 2010; 363(18):1693-1703.

[13] Camidge DR, Bang YJ, Kwak EL, lafrate AJ, Varella-Garcia M, et al. Activity and safety of crizotinib in patients with ALK-positive non-smallcell lung cancer: Updated results from a phase 1 study. Lancet Oncol. 2010; 13(10):1011-1019.

[14] Kim DW, Ahn MJ, Shi Y, De Pas TM, Yang PC, et al. Results of a global phase II study with crizotinib in advanced ALK-positive non-small cell lung cancer (NSCLC). J Clin Oncol. 2012; 30(Suppl): Abstract 7533.

[15] Shaw AT, Kim DW, Nakagawa K, Seto T, Crino L, et al. Crizotinib versus chemotherapy in advanced ALK-positive lung cancer. N Engl J Med. 2013; 368(25):2385-2394.

[16] Solomon BJ, Mok T, Kim DW, Wu YL, Nakagawa K, et al. First-line crizotinib versus chemotherapy in ALK-positive lung cancer. N Engl J Med. 2014; 371(23):2167-2177.

[17] Eberhardt WE, De Ruysscher D, Weder W, Le Péchoux C, De Leyn P, et al. 2nd ESMO consensus conference in lung cancer: Locally advanced stage III non-small-cell lung cancer. Ann Oncol. 2015; 26(8):1573-88.

[18] Schaake EE, Kappers I, Codrington HE, Valdés Olmos RA, Teertstra $\mathrm{H}$, et al. Tumor response and toxicity of neoadjuvant erlotinib in patients with early-stage non-small-cell lung cancer.J Clin Oncol. 2012; 30(22):2731-2738.

[19] Lara-Guerra H, Waddell TK, Salvarrey MA, Joshua AM, Chung CT, et al. Phase II study of preoperative gefitinib in clinical stage I non-small-cell lung cancer. J Clin Oncol. 2009; 27(36):6229-6236.

[20] Rizvi NA, Rusch V, Pao W, Chaft JE, Ladanyi M, et al. Molecular characteristics predict clinical outcomes: Prospective trial correlating response to the EGFR tyrosine kinase inhibitor gefitinib with the presence of sensitizing mutations in the tyrosine binding domain of the EGFR gene. Clin Cancer Res. 2011; 17(10):3500-3506.

[21] Shinichirou I, Nobumasa T, Tomohiko I, Katsumi M, Eishin $\mathrm{H}$, et al. Neoadjuvant Crizotinib Administration and Surgical Resection of Lung Cancer Associated with EML4/ALK Gene Translocation. Haigan. 2015; 55(6):1019-1023. 